Entre el mar, el puerto y la ciudad. Comunidades marítimas urbanas en transición: el caso de la Barceloneta en el siglo XIX Eduard PAGE CAMPOS

Avances del Cesor, V. XVI, No 21, diciembre 2019, pp. 131-160.

ISSNe 2422-6580 / ISSN 1514-3899 - http://web2.rosario-conicet.gov.ar/ojs/index.php/AvancesCesor/index

\title{
Entre el mar, el puerto y la ciudad. Comunidades marítimas urbanas en transición: el caso de la Barceloneta en el siglo XIX
}

\author{
Between the sea, the port and the city. \\ Urban maritime communities in transition: \\ the case of Barceloneta in the 19th century*
}

\author{
Eduard Page Campos \\ Universidad de Barcelona \\ edupage4@hotmail.com
}

(España)

\begin{abstract}
Resumen
El proceso de formación de la Barceloneta explica en buena medida el carácter marítimo de un barrio situado inmediatamente tras el puerto y apartado de la ciudad amurallada. En este artículo abordamos el estudio de la Barceloneta en el siglo XIX a la luz de las propuestas teóricas y conceptuales que la historiografía ha desarrollado para el estudio de aquellas sociedades que viven de su relación con el mar, en especial en el ámbito urbano. A continuación, definimos la situación de la Barceloneta a mediados del siglo XIX como el de una comunidad marítima urbana consolidada, lugar de residencia de los trabajadores marítimos y portuarios y espacio predilecto de las actividades económicas ligadas a la construcción naval y al aprovisionamiento de buques. Asimismo, trazamos un perfil sociodemográfico de la Barceloneta en la segunda mitad del siglo, lo que nos permite definirla como un espacio urbano precarizado, con elevados y crecientes tasas de mortalidad y con una estructura social muy joven y ligeramente masculinizada respecto a la ciudad. Finalmente, estudiamos la mirada que las élites y autoridades urbanas desarrollaron sobre el barrio, estrechamente asociada a preocupaciones higiénicas y morales sobre un espacio social percibido como disruptivo.
\end{abstract}

Palabras Clave: Barceloneta, industrialización, historiografía marítima, demografía urbana, higienismo.

*. Este trabajo ha recibido el apoyo financiero y forma parte del proyecto de investigación del European Research Council "SeaLiT. Seafaring Lives in transition. Mediterranean Maritime Labour and Shipping during Globalization, 1850s-1920s" ERC-2016STG 714437 y del proyecto del Ministerio de Ciencia e Innovación "Mundos del trabajo en transición (1750-1930): cualificación, movilidad y desigualdades" HAR2017-84030-P. 


\begin{abstract}
The formation process of the district of Barceloneta explains, to a large extent, the maritime character of a neighborhood located immediately behind the port and separated from the walled city. In this article we address the study of Barceloneta in the 19th century in light of the theoretical and conceptual proposals that historiography has developed for the study of those societies that live from their relationship with the sea, especially in the urban sphere. Thereupon, we define the situation of Barceloneta in the mid-19th century as that of a consolidated urban maritime community, a place of residence for maritime and port workers and the favored area for the economic activities related to shipbuilding and shipping supplies. We also draw a socio-demographic profile of Barceloneta in the second half of the century, which allows us to define it as a precarious urban space, with high and increasing mortality rates and a very young social structure, slightly masculinized in relation to the city. Finally, we study the gaze that urban elites and authorities developed towards the neighborhood, which was closely associated with hygienic and moral concerns about this social space as it was perceived as disruptive.
\end{abstract}

Keywords: Barceloneta, industrialization, maritime historiography, urban demography, hygienism.

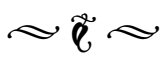

\section{Introducción}

Hay poblaciones esencialmente marítimas, que viven de su propia actividad comercial y mercantil, sin que los grandes elementos de riqueza que proporcionan la agricultura y la industria fabril y manufacturera, coadyuven á su acrecentamiento directo ni formen parte de los diversos ramos de prosperidad y desarrollo de los pueblos.

Estos pueblos están relacionados con estrecho vínculo con todas las plazas mercantiles y marítimas del Universo; la distancia de los mares no es obstáculo á su vecindad, que acorta, estrecha y confunde la actividad humana: y así, el chino, como el yankee; el peruviano, como el persa, el bretón, como el indostano forman la gran familia comercial que han creado las necesidades de las naciones, y que fomentan los respectivos gobiernos con las sabias leyes que arrancan de la base del derecho de gentes.

E1 cosmopolitismo imprime un carácter especial a sus mercados, á sus costumbres, á sus aspiraciones financieras y á sus ideas económico-políticas. ${ }^{1}$

Una pieza de un periódico local, en el año 1880, trazaba con remarcable lucidez los rasgos diferenciales que imprimen sobre la naturaleza de una población sus estrechos vínculos con el mar y el comercio marítimo. Esta introducción, de hecho, servía para caracterizar el barrio marítimo de Barcelona, la Barceloneta. Así seguía la pieza:

1. Los intereses de la Barceloneta (5 de diciembre de 1880). La Unión, revista semanal de intereses morales y materiales de S. Martin de Provensals, Gracia, S. Andrés de Palomar y Barceloneta, p. 1. Arxiu Històric de la Ciutat de Barcelona (AHCB), Barcelona. 
Los productos de su industria están inciertamente enlazadas con los del comercio marítimo, que es la principal fuerza de su riqueza; su comercio está en razón directa del desarrollo de la marina mercante, del cambio de ciertos productos, de las condiciones [de] sus mercados, de la seguridad de sus puertos, de la comodidad de sus depósitos y almacenes, de su fácil comunicación terrestre y de la salubridad de su clima.

Estos pueblos abundan en el litoral de los mares españoles, y el prototipo de estos centros de comercio podemos afirmar que es la Barceloneta, sin que intentemos aminorar la importancia de otras plazas; la Barceloneta, que por la actividad de sus industriales, por la probidad de sus comerciantes, por la inteligencia de sus navieros, armadores y patrones, ha sabido elevarse en importancia á un grado en que puede competir con ventaja con las mejores plazas marítimas de las costas de levante. ${ }^{2}$

La Barceloneta, desde su fundación a mediados del siglo XVIII hasta la actualidad, ha sido considerada como el suburbio o arrabal marítimo de Barcelona, como su distrito portuario, también como la población de Marina o como el barrio de pescadores o de la gente de mar de la ciudad. El punto de vista a través del cual se la ha definido ha variado a lo largo de los años, así como lo ha hecho $\mathrm{su}$ encuadramiento administrativo. Como en el caso del artículo citado, sin embargo, se ha mantenido intacta la consideración de su carácter marítimo y portuario como su principal elemento constituyente.

2. Los intereses de la Barceloneta (5 de diciembre de 1880). La Unión, revista semanal de intereses morales y materiales de S. Martin de Provensals, Gracia, S. Andrés de Palomar y Barceloneta, p. 1. AHCB, Barcelona.
La idea según la cual las poblaciones marítimas tienen una naturaleza social distinta respecto aquellas poblaciones no costeras ha sido objeto de reflexión, más recientemente, dentro de la historia marítima y social. Se han desarrollado así diferentes propuestas conceptuales y teóricas para entender los elementos definitorios de estas sociedades y abordar su estudio histórico. A la luz de estas propuestas, de las cuales pretendemos hacer un pequeño esbozo -especialmente de aquellas concernientes a los barrios marítimos de ciudades portuarias europeas del siglo XIX, nos planteamos comprender la naturaleza como comunidad humana y como espacio urbano de la Barceloneta y, asimismo, su transformación a lo largo del siglo XIX en el contexto del proceso industrializador que experimenta la ciudad y la economía marítima.

Esta investigación se ha realizado a través del trabajo con un variado rango de fuentes. Para el análisis sociodemográfico de la Barceloneta, se han analizado fuentes como el Registro de Defunciones de Barcelona, los censos de población o los Anuarios Estadísticos de la ciudad, lo que se ha combinado con la consulta de las informaciones y estadísticas ofrecidas por diferentes comentaristas sociales contemporáneos al periodo estudiado. Asimismo, para los restantes aspectos tratados en el artículo, se han examinado diferentes guías comerciales de la ciudad y se han consultado algunos periódicos locales y los fondos administrativos de aquellas autoridades bajo las que se encuadraba la Barceloneta en el siglo XIX, en especial la Diputación y el Ayuntamiento de Barcelona. 


\section{La noción de comunidades marítimas}

El comercio marítimo, como expone la cita que encabeza el artículo, ha jugado un papel central en el desarrollo de las sociedades modernas, y los puertos han sido ejes nodales de él y, al mismo tiempo, espacios generadores de dinámicas sociales y culturales específicas. El transporte no ha sido, no obstante, el único medio de vida ligado al mar a través del cual se han configurado diferentes comunidades humanas a lo largo de la historia. La noción de comunidades marítimas surge como respuesta a la necesidad de definir a toda comunidad cuya vida depende de su relación con el mar, fuese esta relación centrada en la explotación pesquera, el marisqueo o la navegación, o una combinación de varias. Esta dependencia configura en estas comunidades unos rasgos sociales particulares. ${ }^{3}$

La idea de comunidad, cabe mencionar, ha experimentado una des-reificación desde su uso extendido en las ciencias sociales en la primera mitad del siglo XX. La comunidad, pues, ya no sólo se entiende como aquella formación social sobre la que aún no han actuado las fuerzas modernizadoras e individualizadoras del cambio social vinculado al desarrollo del comercio y la industria. ${ }^{4} \mathrm{~A}$ pesar de lo complejo de la noción de comunidad, pocos estudios sobre comunidades marítimas han abordado

3. En su estudio de diversas comunidades marítimas de Nueva Inglaterra, Heyrman (1984) muestra como en dichas comunidades, caracterizadas por una mayor apertura comercial, se desarrollaron vínculos e instituciones sociales diferentes de aquellas de interior cercanas a las mismas.

4. Una reflexión sugerente sobre la comunidad como espacio social, y no como concepto opuesto al de sociedad, en Faue (2000).

134 una definición específica del concepto. Hanna Hagmark-Cooper (2003), en su estudio sobre las mujeres de las comunidades de las islas Åland, sí desarrolla una definición que se fundamenta en dos polos. De este modo, existen comunidades con lo que denomina ambiente maritimo, es decir, que tienen una dependencia directa del mar para su existencia, para su sustento y desarrollo material. E1 segundo elemento que sugiere HagmarkCooper es el de la percepción marítima, la idea del mar como eje relevante en la imagen y la identidad del grupo.

Amélia Polónia (2000) desarrolla con mayor detalle un modelo propositivo para el estudio de comunidades marítimas en el que, como en Hagmark-Cooper, se parte de la conjunción de dos esferas complementarias. Polónia diferencia así entre el estudio social de estas poblaciones -sus rasgos económicos, demográficos o políticos- y el estudio comunitario -ligado a los elementos culturales y de identidad, a los mecanismos de integración y exclusión y a las percepciones de las poblaciones en cuestión-. Polónia, de hecho, establece algunos elementos que han de permitir hablar de la existencia de una identidad propia que configura la comunidad. Entre estos elementos se encuentran que la vivencia de grupo se proyecte en experiencias familiares y sociales diferenciales, que la relación con el mar conforme una psicología colectiva que establezca una estructura de valores y un marco referencial propio y que esta conciencia grupal se proyecte socialmente a nivel organizativo. Por otra parte, en dichas comunidades se observan ciertos parámetros demográficos diferenciales. Estas suelen depender de una fuerte movilidad poblacional $y$ de intensos movimientos migratorios, generando fenómenos, en especial en grupos 
ligados a la navegación, como el desarraigo de células familiares amplias y su nuclearización. Las mujeres, debido la falta de estabilidad en el universo masculino, se convierten en los referentes de la identidad social de la comunidad.

La limitación del desarrollo conceptual de la idea de comunidad marítima ha conducido a que su utilización se haya circunscrito a poblaciones de tamaño relativamente reducido, a menudo pequeñas islas y municipios litorales en entornos rurales. Son entidades con un relativo aislamiento geográfico que han configurado comunidades socialmente cerradas, donde los rasgos mencionados suelen encajar. Como la misma Polónia (2000) asegura, conforme estas comunidades se insertan en realidades urbanas mayores, estos fenómenos tienden a disolverse (pp. 21-22). A pesar de ello, el valor del concepto resulta ineludible $y$, entendemos, recuperable para un marco urbano si tenemos en cuenta la disparidad del contexto. Hasta el momento, sin embargo, para el estudio de grupos situados en entornos urbanos, o directamente para las mismas urbes costeras, la historiografía ha recorrido a marcos conceptuales diferentes.

\section{Las ciudades portuarias como modelo}

El principal concepto con el que se ha trabajado desde la interacción entre la historia marítima y la historia urbana es el de ciudades portuarias. El concepto surge inicialmente desde un paradigma funcional-estructuralista, a partir del cual se entienden los rasgos que presentan dichas urbes como genéricos y sujetos a modelizarse. Broeze (1989), de hecho, defiende que este objeto de estudio debe convertirse en un arquetipo con voluntad de aplicación universal (p. 9). En el estudio de las ciudades portuarias, el puerto se entiende como la fuerza central de la ciudad. La historia cuantitativa, así como la historia comparada, se sitúan, en este marco, como instrumentos eficaces para lograr una comprensión de las estructuras comunes de todas las ciudades portuarias, priorizando las analogías mutuas por encima de las particularidades propias (Broeze, 1985 y 1989; Reeves, Broeze y McPherson, 1989).

Dentro de esta propuesta se concibe como elemento definitorio de la ciudad portuaria el hecho que su actividad económica se concentra en el puerto y sus prolongaciones y que esta actividad portuaria se dispone como el principio organizativo de todo el complejo urbano. Las específicas funciones económicas de la ciudad portuaria generan las características diferenciales de esta tipología de ciudad. Es su naturaleza económica, por lo tanto, la que define, para estas ciudades, características y grupos sociales específicos, formas $y$ expresiones culturales propias $y$ un determinado entramado institucional (Konvitz, 1993; Martínez Shaw, 1997).

Para las coordenadas de espacio y tiempo en que se sitúa nuestro objeto de estudio, es decir, para la Europa en proceso de industrialización del siglo XIX, las ciudades portuarias presentan problemáticas concretas. Por una parte, el impacto de la introducción del vapor en la navegación, juntamente con la aplicación del hierro y el acero como material constructivo y la generalización de las hélices, y por la otra parte el crecimiento extraordinario del comercio marítimo fruto de su mundialización forman el contexto general 
en el que se inscribe la historia de las ciudades portuarias en el período. A estos dos elementos se une la enorme movilización de recursos que supuso la modernización de los puertos para su adecuación a esas nuevas realidades. La conjunción de estos fenómenos habría generado impactos sociales y económicos similares a todas las ciudades portuarias (Konvitz, 1994; Osterhammel, 2015). Lo mismo sucede en cuanto a su desarrollo demográfico. El crecimiento poblacional de estas ciudades a lo largo del Ochocientos fue, en general, mayor que el de las medias urbanas estatales. Dicho crecimiento se fundamentaba en unos flujos migratorios constantes y a gran escala, que compensaban crecimientos vegetativos muy tímidos o incluso negativos, resultantes de unas tasas de mortalidad extraordinariamente elevadas (Lawton y Lee, 2002). ${ }^{5}$

La alta mortalidad, de hecho, se sitúa como uno de los rasgos diferenciales por excelencia de las ciudades portuarias, debido a la conjunción de una mayor exposición a las enfermedades epidémicas y unas condiciones sociales precarias -predominio del trabajo casual y mal retribuido, elevada aglomeración urbana, pésimas condiciones habitacionales y acceso segregado a las mejoras higiénicas-. La mortalidad de niños e infantes, que suponía alrededor de la mitad del total de fallecimientos de las ciudades portuarias británicas en la segunda mitad del siglo XIX, tenía una incidencia singular, así como la mortalidad masculina entre adultos, en especial sobre marineros y trabajadores portuarios. La nupcialidad se veía coartada por

5. En Barcelona, entre los años 1833 y 1897, el crecimiento vegetativo fue, de hecho, negativo, y su notable crecimiento poblacional se debió exclusivamente al aporte migratorio (López Guallar, 1995; Tatjer, 1995).

136 unas ratios sexuales desequilibradas en favor de los hombres, resultado del componente masculino del fenómeno migratorio y las oportunidades laborales que ofrecían las estructuras económicas de estas ciudades. Las tasas de fertilidad y natalidad, sin embargo, eran elevadas, animadas por la precariedad de las condiciones sociales existentes (Lawton y Lee, 2002; Lee y Lee, 1998).

\section{Las fronteras del colectivo: marineros, gente de mar y comunidades portuarias}

E1 encuadre de los primeros estudios sobre ciudades portuarias en un marco historiográfico definido por la preponderancia de los factores geográficos y económicos ha dado paso, en años recientes, a la apertura hacia nuevos intereses y metodologías que, junto con la demografía histórica, han permitido redefinir el marco en el cual se desarrollan los estudios sobre dichas ciudades y sobre las comunidades marítimas que las habitan. La incorporación de la dimensión humana ha posibilitado profundizar en las vidas de las personas y grupos humanos que constituyen la ciudad y situarlas en las coordenadas del contexto social urbano en que se desarrollan. ${ }^{6}$

En este nuevo marco se han llevado a cabo investigaciones sobre la identidad de las ciudades portuarias, su forja transecular y el

6. Esta incorporación fue teorizada por Monge Martínez (1998) que, criticando el molde positivista de los estudios sobre ciudades portuarias desarrollados hasta entonces, proponía concebir el puerto como comunidad humana, como el espacio de vida de diferentes colectivos. Más recientemente, ya en un contexto historiográfico distinto, Byrne (2016, p. 245) sugería la definición de la ciudad portuaria como "a network of meaningul lived spaces". 
significado de lo que supone ser de mar para una población. ${ }^{7}$ Los estudios sobre la vida de los colectivos de marineros y de gente de mar dentro de contextos urbanos han tomado, sin embargo, una mayor preponderancia. Esto ha permitido abordar temas como la marginalización y alteridad de estas comunidades, su resistencia a la hegemonía cultural burguesa, el papel que desarrollan en la imaginería nacional, su religiosidad popular alternativa o la percepción que las élites urbanas tienen sobre ellas. ${ }^{8}$

La primera disyuntiva que se presenta a la hora de abordar esta materia es la definición de los límites del grupo humano a estudiar, así como la propia denominación de dicho grupo, la cual varía en función, entre otros, del ámbito lingüístico y nacional. En el caso de la historiografía francesa, las fronteras de la categoría de gente de mar se han alternado entre definiciones más amplias y más estrechas. ${ }^{9}$ En su estudio sobre los marinos de Le Havre, Nicolas Cochard (2016) parte de la definición jurídica de marinos para incluir a todos ellos, junto a sus familias, en la categoría. ${ }^{10}$ Así, todo aquél que trabaja por y para el mar de forma relativamente directa formaría parte

7. Véase, por ejemplo, Carbonell, Doñate y Romero (2011) y Tanis (2016).

8. En esta redefinición ha destacado el grupo Port Towns and Urban Cultures de la Universidad de Portsmouth. Veáse Beaven, Bell y James (2016).

9. El concepto de gente de mar también ha sido de uso generalizado en la historiografía española, habitualmente asociada en el periodo que nos concierne a los trabajadores marítimos que formaban parte de las Matrículas de Mar (García Domingo, 2017; Mateo, 2004b).

10. Se considera marino todo aquél inscrito en la Inscripción Marítima, es decir: pescadores, marineros de altura y de cabotaje, pilotos, constructores navales, etc. del colectivo. Alain Cabantous (2007), por su parte, lo matiza y reduce la noción a aquellos dedicados a la navegación y sus familias, a la vez que advierte sobre la porosidad de las fronteras definitorias del colectivo y de su propia diversidad interna. Así, fenómenos como la pluriactividad plantean unos límites razonados a una categoría cerrada, al mismo tiempo que las diferencias sociales dentro de la comunidad y la correspondiente intersección de la cultura burguesa y la popular con la cultura marítima y su potencial efecto disgregador sobre esta última deben ser tenidas en cuenta. Cabantous previene, por lo tanto, de los peligros de extremar la diferenciación entre la gente de mar y su historia y la del resto de la población.

Otra propuesta que también limita las fronteras del colectivo a estudiar, en este caso en un sentido social vertical, es la que plantea el Grupo de Pesquisa Portos e Cidades no Mundo Atlântico de Rio de Janeiro con el concepto de comunidad social portuaria. Dicha comunidad, arguyen

é composta pelo homem simples, aquele que vive esta realidade complexa e que vaga pelas suas ruas e locais de trabalho, sem dignificação social, que tem como referências as atividades diretas ou indiretas ligadas ao cotidiano e que vive em determinados lugares, marcadamente de pobreza e da exclusão social (Honorato, de Oliveira y Mantuano, 2016, p. 472).

Así, la comunidad portuaria urbana no incluye los elementos más elevados de la escala social ligados a la economía marítima y se vincula a la realidad del espacio urbano segregado en que desarrollan su vida cotidiana los trabajadores portuarios.

La necesidad de insertar al marinero dentro de su contexto urbano y comunitario es lo 
que conduce a Robert Lee (2013) a criticar la noción de la existencia de una cultura marítima específica ligada únicamente al sector ocupacional de la marinería. En contraposición a Andrew Gritt (2005), que defiende la especificidad social de los marineros en las ciudades y su separación física del resto de la población, Lee (2013) remarca tanto las similitudes culturales de los marineros con los trabajadores portuarios terrestres como la pluralidad ocupacional del propio colectivo. Es por ello que entendemos que hay que analizar la comunidad marítima urbana en su contexto vivencial, eso es, considerando a los trabajadores ligados a la economía marítima y portuaria en un sentido amplio y no restrictivo, debido a la alta transferibilidad ocupacional dentro de ella, ${ }^{11}$ a sus fuertes vínculos culturales y al hecho de tener una geografía social dentro de la ciudad prácticamente idéntica. Además de los propios trabajadores, también se inserta en dicha comunidad sus respectivas familias $y$ vecinos, que juegan un papel central en la dinámica comunitaria. Por eso entendemos que la noción de comunidad que debemos preferentemente atender es la que incorpora en un sentido extenso estos estratos sociales ligados a la economía marítima y portuaria y que están delimitados verticalmente por la progresiva inserción de buena parte de la élite del sector en los espacios físicos y culturales de la burguesía urbana.

11. La alta temporalidad de varios de los subsectores de este ámbito económico marcaba la necesidad de ocuparse en otros oficios. Así es el caso, por ejemplo, de los pescadores en época invernal. Asimismo, la corta duración media de las carreras profesionales de los marineros de altura, en otro sentido, forzaba también esta alta transferibilidad que permeaba los diferentes grupos profesionales de la ciudad portuaria.

\section{Reducción de escala y contexto vivencial: hacia la espacialización del objeto de estudio}

En la historiografía anglosajona se ha utilizado con cierta frecuencia el concepto de sailortown para referirse a barrios marítimos de grandes ciudades. ${ }^{12}$ En los estudios desarrollados bajo este concepto se pone el énfasis en la subversión de las convenciones morales burguesas que se producían en dichos espacios por parte de las clases marineras y su percepción por parte de las élites y autoridades locales como lugares de vicio y perdición debido a la presencia de establecimientos de ocio y a los problemas asociados con la bebida y la prostitución. ${ }^{13}$

El concepto, a nuestro entender, reduce un espacio social urbano heterogéneo a un espacio exclusivamente de marineros, ocultando a las mujeres y a los otros trabajadores de la economía marítima y portuaria que convivían con ellos. A pesar de ello, el camino iniciado por estos estudios sobre estos espacios urbanos permite avanzar hacia una mayor concreción espacial de las investigaciones. Esta mayor concisión ha permitido, en diferentes casos,

12. Aunque también para hacerlo con las propias ciudades. Es el caso, por ejemplo, de ciudades sedes de la Royal Navy y de sus astilleros, como Portsmouth y Plymouth.

13. Así, entre las definiciones planteadas nos encontramos con la siguiente: "Sailortowns were the districts of merchant and naval ports where sailors visited, often lived, and were entertained. It was a distinct area characterised by its public houses, brothels, and low entertainment that employed significant numbers of working people" (Beaven, 2016a, p. 160). Especialmente estimulante resulta la transformación de la percepción del espacio urbano ocupado por los marineros a raíz de la transición de la vela al vapor, asociada asimismo a un cambio en negativo de la imagen prototípica del marinero por parte de las élites (Beaven, 2016a, 2016b). 
atender con mayor grado de detalle aspectos concretos de estas comunidades.

Estos estudios han dado testimonio de las condiciones de vida especialmente degradadas de los espacios portuarios, marcados por un régimen laboral caracterizado por trabajos de poca cualificación y alta temporalidad e inestabilidad, debidos en buena medida a la naturaleza discontinua y eventual de la pesca, la navegación y los trabajos de estiba. Asimismo, en dichas zonas se perciben unas peculiaridades demográficas acentuadas respecto a las medias de sus respectivas ciudades: mayor mortalidad y mortalidad infantil, acrecentado desequilibrio entre sexos, retraso de la nupcialidad y mantenimiento de altas tasas de fertilidad, con un consiguiente retraso temporal de la transición demográfica respecto a la cronología dominante en la Europa urbana del Ochocientos (Cochard, 2016; Gibb y Lee, 2002; Lawton, 2002; Oustric, 2016). En otro ámbito, y al amparo de las especificidades de la vida portuaria, se desarrollaron formas y espacios de sociabilidad propios y específicos alejados de los patrones culturales dominantes de la clase media urbana (Beaven, 2016b; Suárez Bosa y Domínguez Prats, 2016). Finalmente, las escasas investigaciones de barrios portuarios específicos parecen evidenciar la tendencia a un relativo aislamiento tanto físico como social de dichos barrios respecto al resto de la ciudad y un grado elevado de cohesión interna y de auto-reconocimiento como grupo de las respectivas comunidades que los componen (Beaven, 2016a; Mateo, 2004a).

A pesar de la importancia que han tenido los estudios sobre ciudades portuarias para lograr una mejor comprensión de la particularidad que, a diferentes niveles, confiere a este tipo de ciudades su vinculación con el mar, resultan insuficientes a la hora de abordar el análisis concreto de las comunidades marítimas que habitan en su seno. En el contexto del siglo XIX, se ha observado la tendencia a la diferenciación de la geografía urbana de los diferentes grupos sociales. ${ }^{14}$ Las comunidades marítimas, en concreto, se encontraban insertadas territorialmente en una zona determinada de la urbe, en la cual las familias y colectivos que vivían de la actividad marítima residían, trabajaban y desarrollaban su vida terrestre, dónde construían sus espacios de sociabilidad y sus redes de amistad, parentesco y solidaridad mutua. Donde, en definitiva, se reducía buena parte del universo material y mental en el que desenvolvían su vida cotidiana.

Situar en el espacio y territorializar el estudio de estas comunidades marítimas en contextos urbanos ha de permitir revalorizar el factor geográfico y enfatizar "la relevancia del dónde, el arraigo material de los hechos históricos en puntos concretos de un espacio activo, vivo y diferenciado" (Oyón y Serra, 2011, p. 388). Asimismo, un enfoque anclado en el espacio en el que se desarrolla la vida de dichas comunidades ha de permitir captar con mayor nitidez los grandes fenómenos sociales que marcan la historia del mundo urbano y del mundo marítimo en el siglo XIX. La focalización en un cuerpo social específicamente localizado ha de permitirnos un entendimiento más detallado de la acelerada urbanización, del fenómeno migratorio y de la densificación poblacional de las ciudades, así como de sus condiciones higiénicas, de la transformación laboral y de la actuación

14. Este fenómeno, en España, es explicado en Otero Carvajal (2007, pp. 258-260). 
desplegada a nivel institucional para abordar estas problemáticas.

La reducción de escala, asimismo, permite una aproximación más íntegra a las comunidades de estudio. Además del imprescindible perfil sociodemográfico de estas, es fundamental el conocimiento de la evolución del puerto al que la comunidad está asociada, así como el de su propia estructura económica y el impacto que en ella ejerce la implementación de la navegación a vapor, la transformación de la construcción naval y las nuevas dimensiones, ritmos y exigencias del comercio marítimo mundializado.Estos fenómenos tienen también un impacto en la estructura ocupacional de la comunidad que debe ser analizado. En paralelo, es imprescindible profundizar el conocimiento sobre el rol de las mujeres, que jugaban un papel central en el sostenimiento de la vida, en la economía marítima y en las dinámicas socializadoras de la comunidad. El mayor localismo del universo cívico de las mujeres contribuyó a que fuesen las principales protagonistas en la formación y consolidación de las redes comunitarias que se vestían en el vecindario, en una escala que anclaba el barrio como principal referente organizativo de las clases trabajadoras (Kaplan, 2003, pp. 178198). Por último, resulta necesario indagar cómo era la percepción que tenían las élites socioeconómicas y culturales de la ciudad de una comunidad localizada en un espacio que era ajeno a su geografía social. La conjugación de estos elementos ha de permitir entender el rol y papel de los barrios y distritos portuarios urbanos en el mundo en transformación del siglo XIX.

Para el estudio de caso que presento se han intentado desarrollar algunas de las principales consideraciones teóricas señaladas hasta ahora. En primer lugar se detalla la formación histórica de la Barceloneta, para analizar a continuación la centralidad del barrio en la economía marítima y portuaria de la ciudad y explicar su estructura social a mediados del siglo XIX. Todo ello me permite definir a la Barceloneta como una comunidad marítima urbana consolidada. Finalmente, se traza su perfil sociodemográfico en la segunda mitad del ochocientos y se estudia la relación de esta comunidad con la mirada e intereses proyectados por las élites sobre ella. Algunos aspectos han quedado fuera del alcance de esta investigación debido a la naturaleza de las fuentes disponibles. Entre estos aspectos destaca, sobre todo, la centralidad de las mujeres en el seno de la comunidad y las estructuras y relaciones de género dominantes en la Barceloneta a lo largo del período.

\section{Génesis y evolución de la Barceloneta entre los siglos XVIII y XIX}

El antiguo barrio de la Ribera era, en la Edad Moderna, el espacio predilecto de residencia de la gente de mar de Barcelona.Tras la derrota de la ciudad en la Guerra de Sucesión, las nuevas autoridades borbónicas ejecutaron su derribo para así poder construir una fortaleza militar, la Ciutadella, que controlaría desde entonces la ciudad. En el año 1716, poco antes del derribo, de las 331 familias barcelonesas con un cabeza de hogar con oficio marítimo, el 74,9 \% vivían en el Quarter de Mar y, específicamente, el $50,8 \%$ en las islas de casas que formaban la Ribera (Garcia Espuche, 2007, pp. 44-45, 52). En aquellas fechas, el espacio sobre el que se construiría la Barceloneta era un arenal 
triangular de reciente formación ${ }^{15}$ situado en el exterior del recinto amurallado de Barcelona e inmediatamente a continuación del único muelle con el que contaba el puerto de la ciudad. Sobre este arenal se hallaban decenas de barracas ocupadas, mayoritariamente, por individuos ligados a los oficios de la mar. A inicios del siglo XVIII, de 110 barracas contabilizadas, 62 eran utilizadas por pescadores y 5 por carpinteros de ribera y calafates (Garcia Espuche, 2007, p. 46). Además de las barracas, que tenían tanto funciones residenciales como de almacén de herramientas de trabajo y de mercaderías portuarias, el arenal era un espacio utilizado para construir y varar barcos y para tejer y reparar redes de pesca. El arenal había crecido como abrigo del puerto de la ciudad, y las propias actividades portuarias habían ido situándose en buen grado a su orilla izquierda. De esta forma, la vinculación entre el puerto y este espacio físico se produjo tempranamente $y$ de forma espontánea.

La fundación del barrio de la Barceloneta sobre este espacio no se produce hasta el año 1753. Los motivos que explican su definitiva construcción, por lo tanto, van más allá de las consecuencias de la Guerra de Sucesión y se insertan plenamente en las dinámicas de la ciudad a mediados del siglo XVIII. Como primer factor se encuentra la propia voluntad de las autoridades de controlar y sanear un espacio que se percibía como peligroso e incontrolado. ${ }^{16}$ En segundo lugar, se sitúa el

15. El inicio de la construcción del puerto en el siglo XV provocó la progresiva acumulación de sedimentos que permitió el avance perpendicular de la línea de mar y la existencia, en consecuencia, del arenal (Alemany, 1998, pp. 56-61).

16. Sebastián Coll, el cronista contemporáneo de la fundación de la Barceloneta, describía las barracas allí instaladas como "unas intrincadas hediondas zahúrdas, crecimiento demográfico que la ciudad vive a lo largo de las décadas posteriores a la guerra de Sucesión. La población barcelonesa se dobló entre los años 1718 y 1748, fenómeno con unos efectos sociales y urbanísticos multiplicados debido a la presión ejercida por las murallas de la ciudad, que evitaban su expansión horizontal. En último lugar nos encontramos con el paralelo crecimiento de la actividad portuaria debido al dinamismo que había adquirido la economía catalana que conformaba el hinterland del puerto. El nuevo barrio se concibió, por lo tanto, para cumplir tres misiones: dar cabida a parte de la población que se aglomeraba en la ciudad, ordenar urbanísticamente un espacio de ocupación parcialmente espontánea y no controlada y acomodar espacios y colectivos ligados a la actividad portuaria. ${ }^{17}$

Las manzanas del nuevo barrio, notablemente alargadas, se dividieron en parcelas cuadradas, dando paso a la configuración a lo largo del barrio del modelo de casa que dominaría por completo: las casas unifamiliares de planta baja y piso. El tamaño de estas parcelas era de 8,4 por 8,4 metros, dando como resultado plantas de $70,56 \mathrm{~m}^{2}$. El área total de las parcelas iniciales contando ambas plantas era, por lo tanto, de 141,12 $\mathrm{m}^{2}$ (Permanyer, 2003, pp. 24-26; Tatjer, 1973, pp. 42-53). La estructura urbanística de la Barceloneta se mantuvo intacta en las primeras décadas del Ochocientos, y todo el crecimiento poblacional se canalizó a través de la expansión horizontal del barrio. Fruto de la presión de los propietarios, en 1838 se dio

en donde parece no era capaz de habitar la hermosura de la virtud, sino el vicio, que como reo busca siempre la confusión de las tinieblas" (Coll i Llopis, 1755, p. 4).

17. El conocimiento de la historia de la Barceloneta hasta hoy en día se la debemos principalmente a los estudios llevados a cabo por Mercè Tatjer $(1973,1988)$. 
Plano 1: Barcelona y el espacio del arenal antes de la construcción de la Barceloneta.

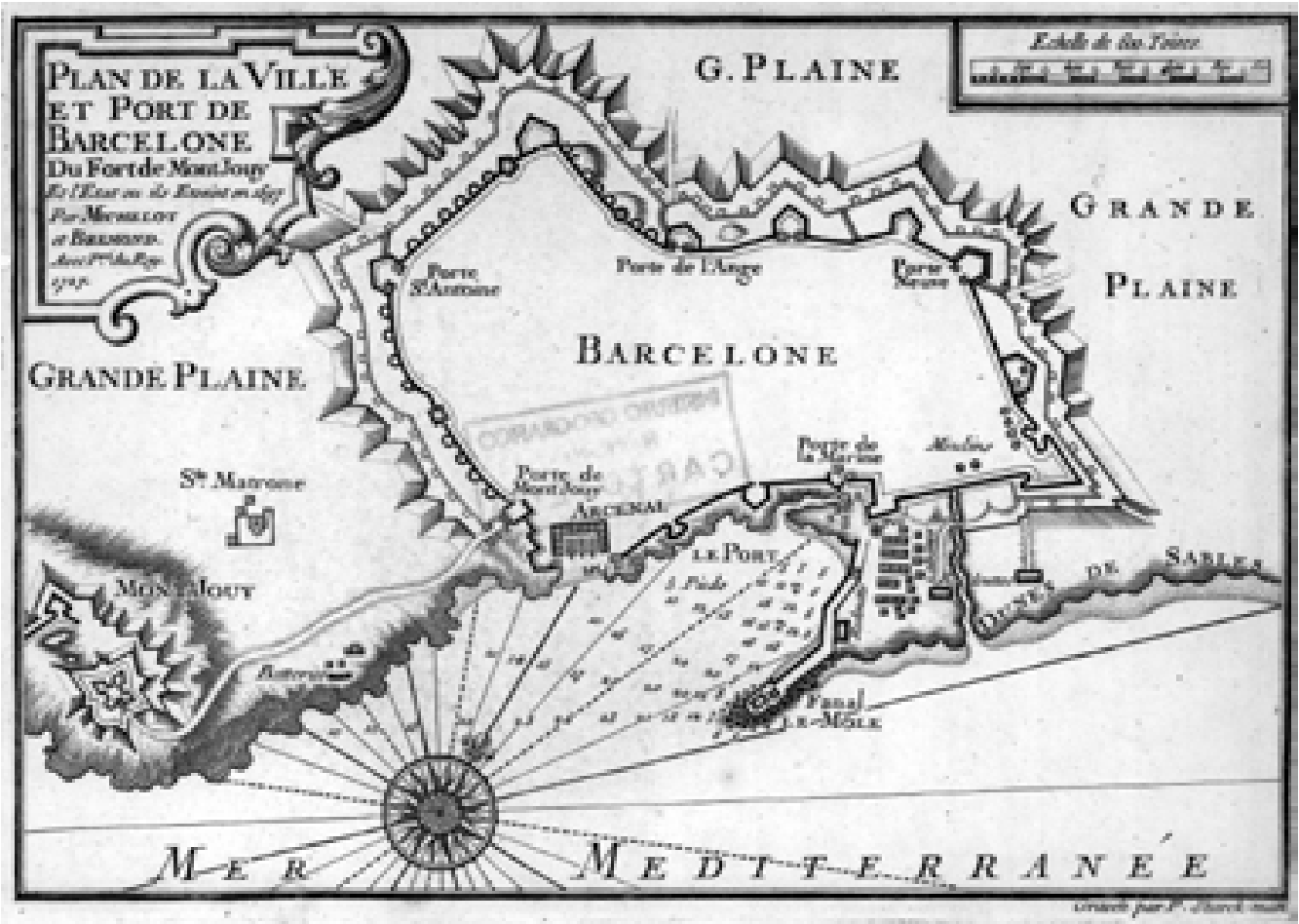

Plan de la Ville et Port de Barcelone.

Du Fort de Mont Jouy Et l'Etat ou ils Etoient en 1697.1727.

Cartoteca del Instituto Geográfico Nacional (INE), signatura 11-C-51.

autorización para levantar un segundo piso, permiso que se repetiría en los años 1868 y 1872 para un tercero y un cuarto, respectivamente. El crecimiento de la población, de este modo, se canalizó a partir del segundo tercio del siglo XIX a través de un crecimiento vertical que multiplicó la densidad habitacional del barrio y que conllevó una progresiva reducción parcelaria. Dicha reducción se llevó a cabo tanto a partir de la separación de las casas entre plantas como por la propia división de estas, dando lugar al tipo parcelario que dominaría el barrio hasta nuestros días: el llamado quart de casa, con un volumen de $35,28 \mathrm{~m}^{2}$ de los cuáles los realmente habitables no solían superar los $30 \mathrm{~m}^{2}$ (Tatjer, 1973, pp. 58-69, 1988, pp. 4657) (véase, planos 1,2 y 3 ). 
Plano 2: Barcelona y la Barceloneta a principios del siglo XIX.

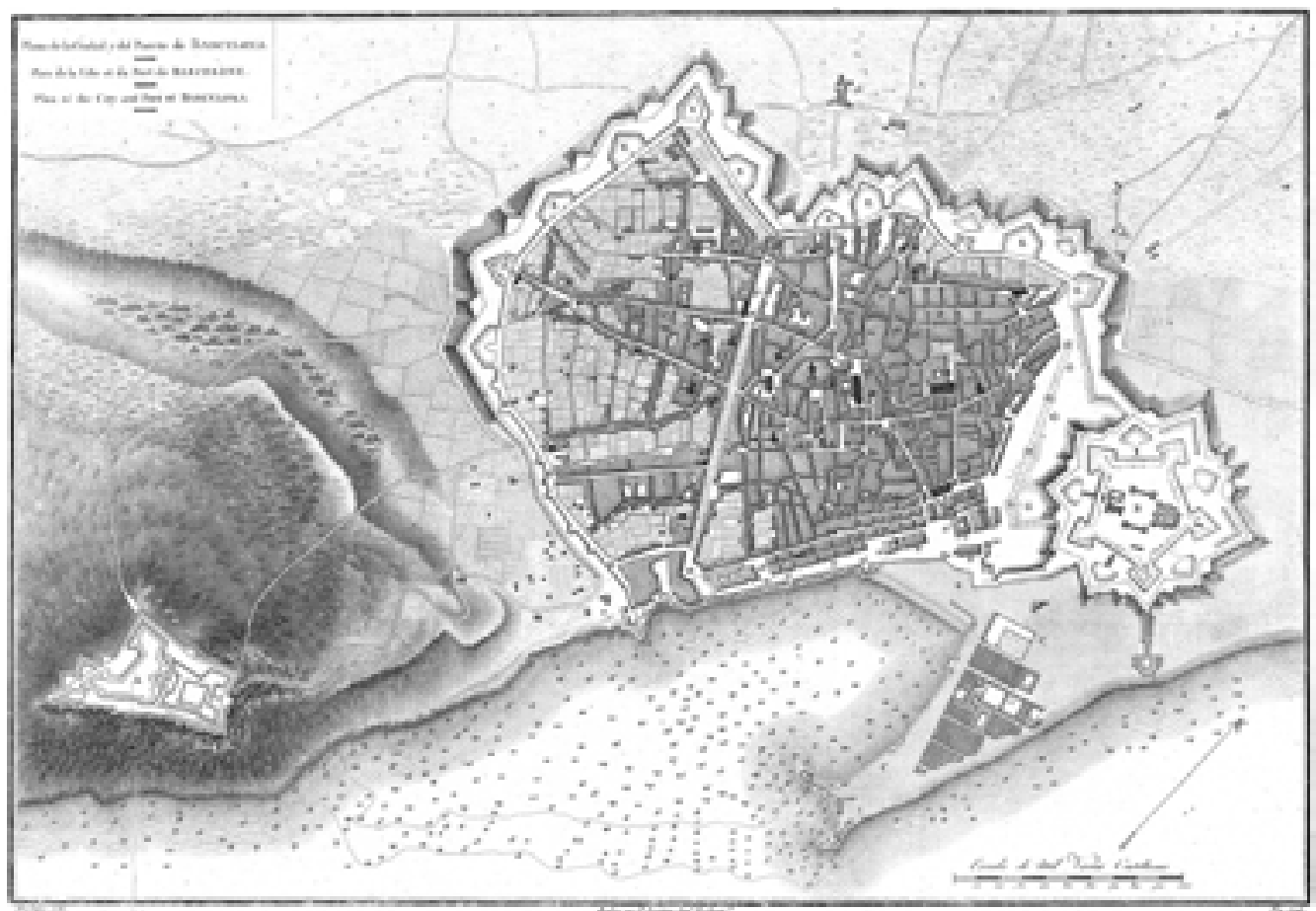

Plano de la Ciudad y del Puerto de Barcelona. 1808.

Cartoteca del Instituto Geográfico Nacional (INE), signatura 11-C-52

\section{La Barceloneta, una comunidad marítima urbana consolidada a mediados del siglo XIX}

Durante el proceso de construcción del barrio, los que tuvieron prioridad para recibir solares en él fueron tanto aquellos que tenían barracas en el arenal como la gente de mar. Como hemos visto, es probable que la coincidencia de nombres entre ambos colectivos fuese elevada. La propiedad del suelo se repartió a través de solares cedidos en forma de plena propiedad por parte de la autoridad militar. Fuentes fiscales de las décadas centrales del
Ochocientos han permitido a Mercè Tatjer desentrañar la composición socioprofesional del colectivo propietario de la Barceloneta. Entre los propietarios que aparecen en el Amarillamiento llevado a cabo entre 1848 y 1849 se observa una presencia abundante de oficios ligados más directamente o menos a la economía marítima, en especial a la construcción naval. Los propietarios de establecimientos comerciales, así como artesanos, industriales y almacenistas tienen también un notable peso (Tatjer, 1988, pp. 7497). Al respecto de esta composición, Tatjer comentaba: 
Plano 3: Barcelona y la Barceloneta a mediados del siglo XIX.

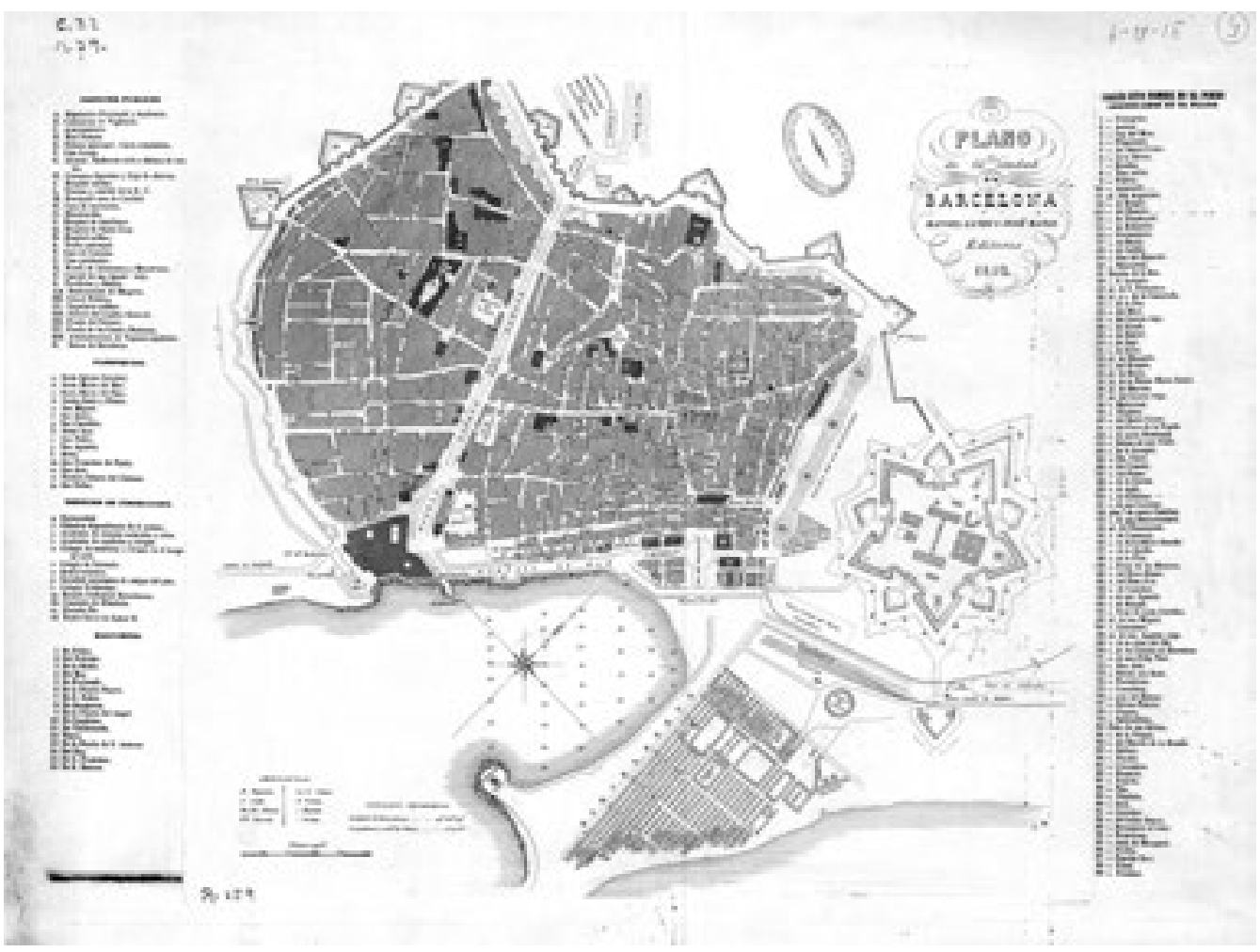

Plano de la Ciudad de Barcelona, 1852. Cartoteca del Instituto Geográfico Nacional (INE), signatura 31-A-9

Elaprovisionamiento y la construcción naval (...) es la base de acumulación que permitirá el surgimiento del bloque de propietarios de la Barceloneta. Los componentes de la "menestralia" de constructores navales ("mestres d'aixa", calafates, corderos, veleros, boteros) enriquecidos por su activa participación en el comercio americano, como proveedores, como inversores en la financiación de embarcaciones e incluso como tripulantes, colocarán parte de sus beneficios en inmuebles en la Barceloneta; tanto como inmuebles de renta ya desde fines del siglo XVIII, y en algún caso como almacenes o uso artesanal (1988, p. 66).

El arquitecto Miquel Garriga i Roca, en su estudio para una reforma urbanística que finalmente no se llevaría a cabo, realizó un censo por oficios de los habitantes del distrito marítimo de la ciudad en el año 1862. Un total de 4719 hombres respondieron a la encuesta 


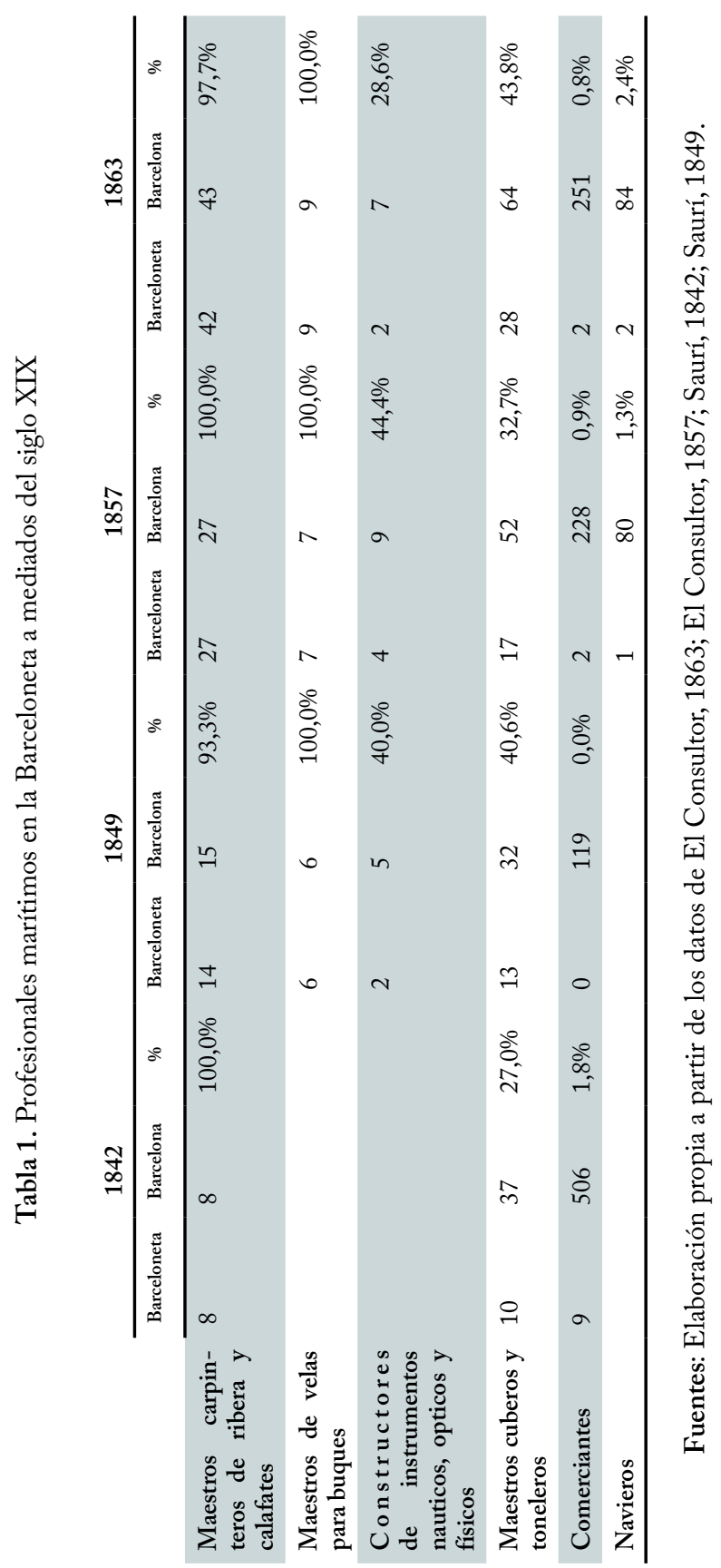

llevada a cabo por el arquitecto. En dicho censo, prácticamente la mitad de los encuestados declaran un oficio directamente vinculado con el mar (47,89\%), a los cuáles se les tiene que añadir una numerosa presencia de otros oficios relacionados: un $11,23 \%$ de jornaleros, de los cuáles buena parte corresponden muy probablemente a trabajadores del muelle; y otras profesiones como carpinteros, fundidores, hojalateros, herreros, maquinistas y toneleros, que tenían una participación fundamental en la construcción y reparación naval y en el aprovisionamiento de buques (Garriga i Roca, 1862, Capítulo 9). ${ }^{18}$

El peso del sector de la construcción naval en el barrio era, de hecho, muy destacado. Los diferentes oficios y establecimientos relacionados más directa o indirectamente con este sector se concentraron en la Barceloneta tempranamente. Los maestros carpinteros de ribera $y$ calafates de la ciudad, directamente encargados de construir y reparar las embarcaciones de madera, se localizaban prácticamente en su totalidad en el barrio. Lo mismo sucedía con los maestros fabricantes de velas para buques. Otros oficios, que colateralmente también contribuían al equipamiento de los

18. A modo de ejemplo, en una localidad mediana del litoral catalán como Sant Feliu de Guíxols, de notable tradición marinera, en el año 1824 el porcentaje de oficios marítimos sobre el total es inferior, rondando el 40\% (Mateo, 2000, pp. 10-11). 
Tabla 2. Concentración de marineros y pescadores en la Barceloneta (1840-1879).

\begin{tabular}{lccc}
\cline { 2 - 4 } Año & $\begin{array}{l}\text { Marineros i pescadores en } \\
\text { la Barceloneta* }\end{array}$ & $\begin{array}{l}\text { Marineros i pescadores } \\
\text { en Barcelona** }\end{array}$ & $\begin{array}{l}\text { Marineros i pescadores en la } \\
\text { Barceloneta respecto al total }\end{array}$ \\
\hline $\mathbf{1 8 4 0}$ & 71 & 92 & $77,17 \%$ \\
$\mathbf{1 8 5 0}$ & 77 & 99 & $77,78 \%$ \\
$\mathbf{1 8 6 0}$ & 125 & 146 & $85,62 \%$ \\
$\mathbf{1 8 6 9 - 7 0 ^ { * * }}$ & 159 & 189 & $84,13 \%$ \\
$\mathbf{1 8 7 9}$ & 169 & 214 & $78,97 \%$ \\
\hline
\end{tabular}

*. Se contabilizan los marineros y pescadores difuntos en cada año y los hijos/as de estos que sean menores de 20 años y soltero/as.

**. De agosto de 1869 a julio 1870. Esta limitación cronológica, debido a la epidemia de fiebre amarilla que afecta la ciudad a partir de agosto de 1870 y altera los datos abruptamente.

Fuente: Elaboración propia a partir del vacíado del Registro de Defunciones, libros correspondientes a los años 1840, 1850, 1860, 1869, 1870 y 1879, Serie A171 de Mortalidad, Arxiu Municipal Contemporani de Barcelona (AMCB), Barcelona.

buques y, por extensión, al negocio marítimo, en especial los toneleros, también tenían una presencia destacada en el distrito marítimo (véase, Tabla 1).

A partir del vaciado documental del Registro de Defunciones del Registro Civil de Barcelona llevado a cabo en el marco de nuestro proyecto de investigación en curso hemos podido comprobar la extraordinaria concentración de marineros y pescadores en el barrio. El porcentaje de registrados en la ciudad con una de estas dos ocupaciones que residen en la Barceloneta siempre es superior, en los años analizados, al 70\% del total, en un período en que la población de la Barceloneta respecto al total de la ciudad se movía entorno al $7 \%$ (véase Tablas 2 y 3 ).

Dicho porcentaje se matiza notablemente cuando hablamos de la cúspide de la jerarquía de las tripulaciones, puesto que de aquellos catalogados como pilotos, navegantes $o$ patrones o capitanes de buque, en los mismos 146 años analizados, residían en la Barceloneta el $14,29 \%$ del total.

La presencia de comerciantes y navieros, por su parte, es anecdótica (Tabla 1). Su baja presencia en el barrio que indican tanto en las guías comerciales de la ciudad como el censo de Garriga i Roca se corrobora en los datos extraídos del Registro de Defunciones, en los cuáles tan sólo el 1,05\% de los oficios anotados entre los residentes del barrio eran comerciantes entre los años 1840 y 1879 (14 individuos de un total de 1338 en los que la profesión viene referida). Eso nos permite corroborar como, a mediados de la centuria, los sectores capitalistas que financiaban las operaciones ligadas a la economía marítima, y sobre los que recaía la mayor parte de los beneficios de la inserción de Barcelona en las redes transoceánicas coloniales, no residían en el barrio, con la única excepción de aquellos vinculados a la construcción naval. ${ }^{19}$

19. La imagen perfilada en la cita que abre el artículo 
Tabla 3. Evolución de la población de la Barceloneta y Barcelona (1848-1900).

\begin{tabular}{lccc}
\multirow{2}{*}{ Año } & \multicolumn{3}{c}{ Población de hecho } \\
\cline { 2 - 4 } & Barceloneta & Barcelona & \% \\
\hline $\mathbf{1 8 4 8}$ & 11808 & 161307 & $7,32 \%$ \\
$\mathbf{1 8 6 0}$ & 14700 & 189948 & $7,74 \%$ \\
$\mathbf{1 8 7 7}$ & 17065 & 249106 & $6,85 \%$ \\
$\mathbf{1 8 8 2}$ & 15802 & 244263 & $6,47 \%$ \\
\hline $\mathbf{1 8 8 7}$ & 17056 & 272481 & $6,26 \%$ \\
$\mathbf{1 9 0 0 *}$ & 20538 & 359986 & $5,71 \%$ \\
\hline
\end{tabular}

*. Del total de población de hecho de Barcelona en 1900 están descontados aquellos habitantes de los pueblos agregados a la ciudad durante la década de 1890.

Fuentes: Elaboración propia a partir de los datos de: 1848: Pi i Arimón, 1854, pp. 301-306; 1860:

Guitert de Cubas, A. (21 de junio de 1896). Barceloneta. Censos de población. El Vigía de la Barceloneta, p. 1. Arxiu Històric de la Ciutat de Barcelona (AHCB), Barcelona; Junta General de Estadística (1863), pp. 80-81; 1877: Guitert de Cubas, A. (21 de junio de 1896). Barceloneta. Censos de población. El Vigia de la Barceloneta, p. 1; Instituto Geográfico y Estadístico, 1879, fig. 1; 1882: Colomer Codina, 1883; 1887: Guitert de Cubas, A. (21 de junio de 1896). Barceloneta. Censos de población. El Vigía de la Barceloneta, p. 1; Anuario estadístico de la ciudad de Barcelona. Año 1902, 1903, p. 97; 1900: Anuario estadístico de la ciudad de Barcelona. Año 1902, 1903, pp. 99-101.

La Barceloneta, a mediados del siglo XIX, presentaba una concentración eminente del colectivo marítimo de la ciudad en este espacio segregado de la ciudad amurallada. La vinculación con la actividad portuaria, dada por la continuidad física entre el muelle y el barrio,

está, por lo tanto, voluntariamente tergiversada. se intensificó con el asentamiento residencial de la gente de mar y con el establecimiento de espacios de trabajo y pequeño comercio ligados a la economía marítima. La Barceloneta era el espacio en el que vivían, trabajaban $\mathrm{y}$ se relacionaban tanto los marineros y pescadores como los trabajadores del muelle y sus respectivas familias. El barrio formaba el principal espacio de referencia social y mental del colectivo, aspecto reforzado por el relativo aislamiento físico de la población que posiblemente multiplicaba la relevancia de las redes de solidaridad vecinales construidas por las mujeres a partir de los principales espacios de socialización barriales. Esta comunidad marítima urbana, no obstante, estaba limitada por una frontera social que excluía a aquellos sobre los que recaían los principales beneficios de la actividad económica ligada al mar y al puerto, los cuáles se encontraban en un espacio físico diferenciado de los trabajadores, artesanos y pequeños comerciantes del sector. La segregación física iba acompañada de una separación de los marcos de referencia en que se desarrollaban sendas experiencias urbanas y que, por lo tanto, trazaban los límites de una comunidad de la cual sus escalafones más elevados se habían autoexcluido.

\section{Un perfil sociodemográfico del barrio, 1840-1900}

La Barceloneta presenta una particularidad remarcable respecto a otros barrios portuarios: su temprana industrialización vinculada a la industria metalúrgica. El asentamiento de estas fábricas, que se situaron preferentemente, entre los años 1830 y 1860, en la zona conocida como la Ginebra -situada en la parte noreste del barrio- hizo que dentro del mismo 
existiera un espacio eminentemente industrial que huía de las lógicas directamente ligadas al mar. A pesar de ello, tres de las cuatro empresas industriales más destacadas de la Barceloneta participaron, en sus inicios, de algunos intentos de hacer aflorar en la ciudad una emergente construcción naval a hierro y vapor. Este intento no se consolidó y, después de escasas construcciones, la nula rentabilidad hizo que se especializasen en construcciones metálicas, máquinas de vapor, puentes o locomotoras, entre otras. Tan sólo la Nueva Vulcano,instalada en el Muelle Nuevo desde la década de los años 1830, se mantuvo como el principal taller de reparación de embarcaciones del puerto. Las demás empresas siguieron colateralmente vinculadas a la economía marítima a través del equipamiento de buques, con la fabricación de vapores, calderas o piezas metálicas (Alfaro Zaforteza, 2007; Moreno Rico, 2011; Riera i Tuèbols, 2008; Tatjer, 1973).

El desarrollo de la Barceloneta a partir de los años '40 del siglo XIX se inserta, de este modo, en la simbiosis entre esta incipiente industrialización ligada al sector metalúrgico y la evolución particular de la economía marítima. Desde los años 1830, muy relacionado al mercado colonial y a las necesidades de la naciente industria textil catalana, el tráfico marítimo en el puerto de Barcelona se multiplicó y todos los sectores vinculados a él se dinamizaron (Carreras y Yáñez, 1992). En paralelo, la transformación tecnológica empezaba a tener un impacto significativo. Tras los iniciales intentos fallidos de desarrollar una industria de la construcción de embarcaciones a vapor, la generalización de la nueva tecnología se produjo en las décadas de 1860 y, sobre todo, de 1870 y 1880, a través de la compra por parte del sector naviero catalán de buques de hierro construidos en el extranjero (Rodrigo Alharilla, 2007; Valdaliso, 2001). Los ritmos de trabajo tanto a bordo como en el muelle, el papel de pilotos y capitanes y las propias categorías laborales se transformaron. La liberalización del trabajo y del comercio, que tuvieron como puntas de lanza la supresión de la Matrícula de $\operatorname{Mar}^{20}$ y del derecho diferencial de bandera, respectivamente, añadieron una nueva dimensión a estos cambios (García Domingo, 2017; Ibarz Gelabert y Romero Marín, 2009; Pascual i Domènech, 1991). También lo hizo la renovación de la infraestructura portuaria, con una ambiciosa reforma que arrancó a inicios de los años 1870 y que permitió al puerto barcelonés disponer de mucha mayor capacidad (Alemany, 1998). Los antedichos fenómenos perfilan el contexto sobre el cual la comunidad marítima urbana de la Barceloneta se desarrolló hasta finales del Ochocientos. A pesar de todas las transformaciones, esta siguió siendo el espacio de concentración primordial de los trabajadores marítimos de la ciudad. Según el censo de población del año 1900, de los 2479 trabajadores en industrias marítimas de la ciudad contabilizados, ${ }^{21} 1980$ residían en la Barceloneta ( Ayuntamiento de Barcelona, 1903, pp. 152-161). Se trata de un 79,87\% del total, cifra muy similar a las que reseñamos para el período entre 1840 y 1879 para marineros y pescadores.

20. Institución, creada por el Estado borbónico en el siglo XVIII, que encuadraba de forma obligatoria a todos los hombres con un oficio marítimo. Todos los matriculados debían realizar el servicio militar en la Armada y estar durante años a su disposición en caso de reclutamiento. A cambio, estos recibían la exclusividad en el ejercicio de sus profesiones (García Domingo, 2017, pp. 33-62; Llovet i Verdura, 1980, pp. 17-43).

21. Dentro de esa categoría se incluyen tanto marineros como trabajadores en la carga y descarga de buques. Sin embargo, no son incluidos los pescadores. 
Tabla 4. Tasas de masculinidad en la Barceloneta y Barcelona (1848-1900)

\begin{tabular}{lll} 
Año & Barceloneta & Barcelona \\
\cline { 2 - 3 } $\mathbf{1 8 4 8}$ & 100,22 & 96,26 \\
$\mathbf{1 8 8 2}$ & 96,84 & 91,85 \\
$\mathbf{1 8 8 7}$ & 98,69 & 94,94 \\
$\mathbf{1 9 0 0}$ & 98,92 & 95,49 \\
\hline
\end{tabular}

Fuentes: Elaboración propia a partir de los datos de: 1848: Pi i Arimón, 1854, pp. 301-306; 1882: Colomer Codina, 1883; 1887: Guitert de Cubas, A. (21 de junio de 1896). Barceloneta. Censos de población. El Vigía de la Barceloneta, p. 1; Anuario estadístico de la ciudad de Barcelona. Año 1902, 1903, p. 97; 1900: Anuario estadístico de la ciudad de Barcelona. Año 1902, 1903, pp. 99-101.

El crecimiento de la población del barrio fue sostenido y prácticamente ininterrumpido, y mientras en los años 1840 la población superaba ligeramente los 10.000 habitantes, en 1900 ya sumaba 20.538 personas en su seno. El crecimiento se concentró, sobre todo, en las décadas centrales del siglo, se estancó ligeramente entre los años 1870 y 1890 y volvió a repuntar en la década final, siguiendo un patrón evolutivo similar al de Barcelona en su conjunto. El incremento fue, sin embargo, inferior al que se produjo en la ciudad, lo cual se tradujo en un ligero descenso del peso del total de la población de la Barceloneta dentro de la urbe (véase Tabla 3).

Uno de los rasgos característicos que se observa en las ciudades portuarias del siglo XIX es una ratio sexual masculinizada debido al peso fundamental de una inmigración joven y masculina. No es el caso de Barcelona, que observa a lo largo del período una presencia más abultada de mujeres que de hombres en su población. ${ }^{22}$ En la Barceloneta sí que se observa, sin embargo, un mayor número relativo de varones con respecto a la ciudad, aún sin ser una sociedad masculinizada (véase, Tabla 4).

La notable inmigración masculina sobre el barrio, juntamente con las escasas oportunidades laborales para las mujeres en la economía formal del puerto, contribuyeron a esta relativa mayor masculinización que en la ciudad.

Otro elemento que observamos en el cuerpo social de la Barceloneta es una estructura por edades muy joven. En 1870, el 45,7\% de los habitantes del barrio tenían 20 años o menos, y el $25,4 \%$ no superaba los 10 años de edad. La diferencia con la estructura general de la ciudad es apreciable, según los datos que nos ofrece el censo de población más cercano cronológicamente. En las mismas franjas de edad, en 1877 un 38,92\% de los habitantes de la ciudad condal no superaban los 20 años, y un 19,98\% los 10 (véase Gráfico 1). Los rasgos particulares del trabajo marítimo y portuario explican, junto con el fenómeno migratorio, una estructura tan joven de la población. Dicha estructura es, también, el reflejo de un retraso destacado de la transición demográfica en la Barceloneta, que se manifiesta, sobre todo, en la permanencia de elevados índices de natalidad.

22. De hecho, la tasa de masculinidad de la ciudad, tanto en el censo de 1877 como en el de 1887, está por debajo al del total de la provincia. Tan sólo en el primer censo español, de 1857, se contabilizan más hombres que mujeres en la ciudad de Barcelona. 
Gráfico 1.1.: Pirámide de población de la Barceloneta en el año 1870

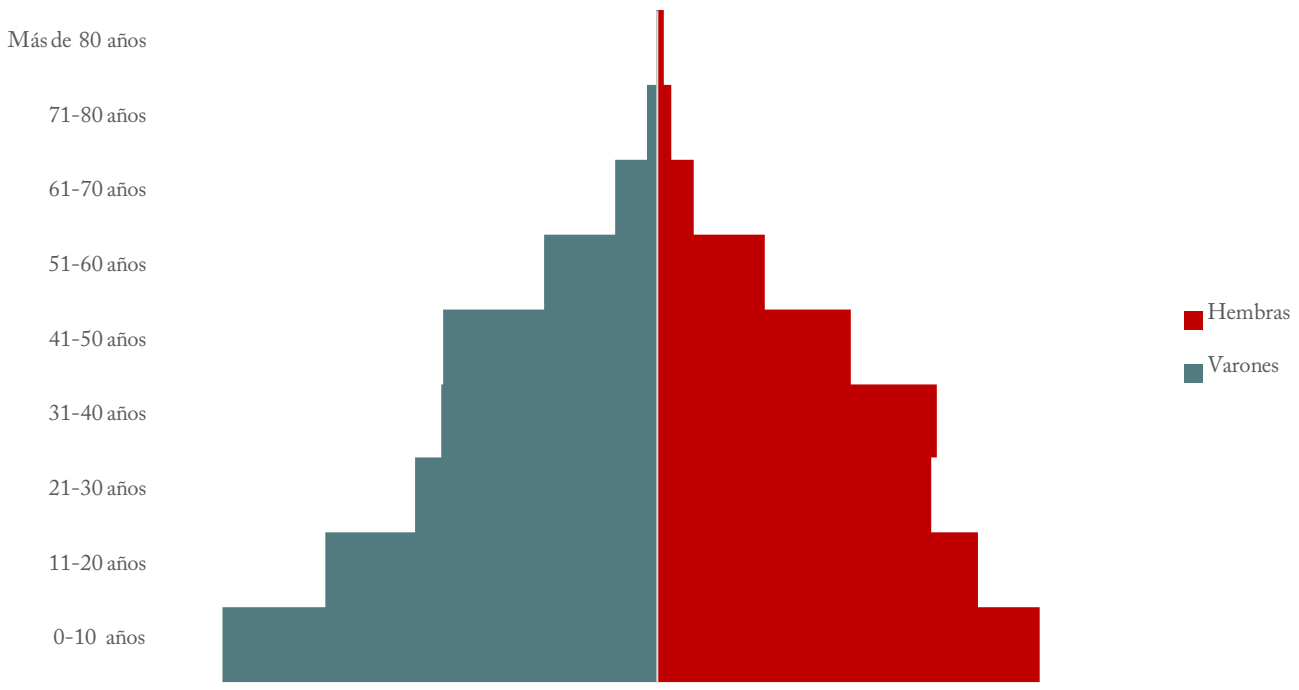

Nota: La pirámide se ha elaborado a partir de una muestra de 1488 individuos, aquellos registrados en el padrón consultado. Supone alrededor del 10\% del total de la población del barrio.

Nota: Debido a las franjas de edad ofrecidas en el Censo de población de 1877, no se han podido equilibrar todas las franjas de la pirámide. Así, la primera barra agrupa 11 años de edad, mientras el resto sólo lo hace con 10 cada una. En el caso de la pirámide de la Barceloneta para 1870, se han adaptado a las mismas franjas adoptada para la de Barcelona en 1870 para permitir la comparación visual.

Fuente: Elaboración propia a partir del vacíado del Padrón de la Conrería y el Asilo de Montalegre, Serie A183 de la Comisión de Gobernación del Ayuntamiento de Barcelona, expediente 4500.15, f. 263-281, 307-320. Arxiu Municipal Contemporani de Barcelona (AMCB), Barcelona.

El retraso en la transición demográfica también es observable en los datos de mortalidad de la comunidad. Las tasas de mortalidad de la Barceloneta tuvieron una tendencia creciente entre mediados $\mathrm{y}$ finales del siglo XIX. ${ }^{23} \mathrm{En}$

23. Algunos problemas metodológicos limitan el conocimiento de las tasas de mortalidad durante todo el periodo: los fallecidos en los hospitales y en las casas de beneficencia solían añadirse a los datos del total de la ciudad, sin ser computables en sus distritos o barrios. Eso 150 el año 1890 la tasa de mortalidad del barrio seguía en niveles sumamente elevados, y no es hasta la última década del siglo XIX cuando los niveles de mortalidad se reducen de forma significativa y permiten hablar del inicio de la transición a unos niveles de mortalidad relativamente equilibrados (véase, Tabla 5).

hacía que las tasas de estos últimos soliesen ser inferiores a los de la ciudad. 


\section{Gráfico 1.1.: Pirámide de población de Barcelona en el año 1877}

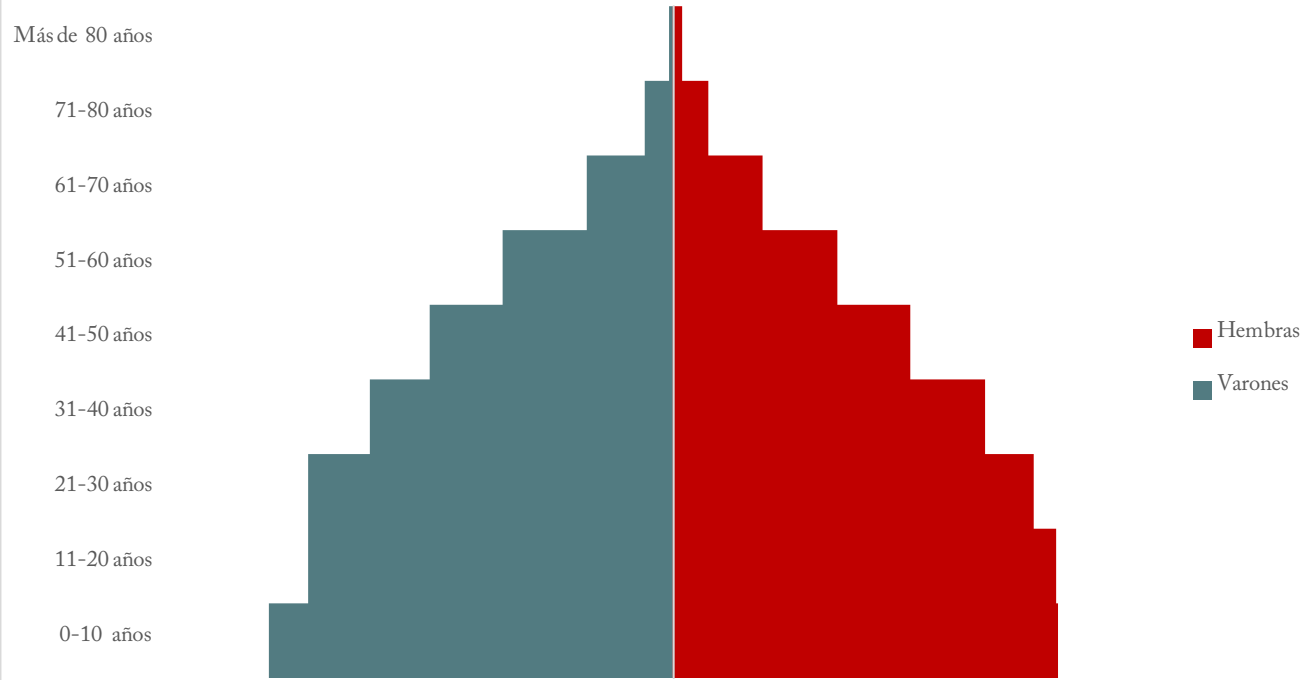

Nota: Debido a las franjas de edad ofrecidas en el Censo de población de 1877, no se han podido equilibrar todas las franjas de la pirámide. Así, la primera barra agrupa 11 años de edad, mientras el resto sólo lo hace con 10 cada una.

Fuente: Elaboración propia a partir de los datos de Anuario estadistico de la ciudad de Barcelona. Año 1902,1903, p. 147.

Tabla 5. Tasas de mortalidad en la Barceloneta $y$ Barcelona (1856-1900)

\begin{tabular}{lll}
\hline Año & Barceloneta & Barcelona \\
\hline $1856-65$ & $27,9 \% 0$ & $25,9 \% 0$ \\
$1876-80$ & $30,8 \% 0$ & \\
$1881-89$ & $33,5 \% 0$ & $30,9 \% 0$ \\
1890 & $32,9 \% 0$ & $30,7 \% 0$ \\
1900 & $25,7 \% 0$ & $25,4 \% 0$ \\
\hline
\end{tabular}

Fuente: Elaboración propia a partir e los datos de: 1856-65: Cerdà, 1868 , p. 549

1876-80: Colomer Codina, 1883; 1881-89: García Faría, 1893, pp. 144-145; 1890 y 1900: Registro de Defunciones, libro correspondiente a los años 1890 y 1900, Archivo del Registro Civil de Barcelona (ARCB), Barcelona; Anuario estadístico de la ciudad de Barcelona. Año 1902, 1903, p. 97; 217.
Aún más revelador supone el diferencial por edades de las mortalidades en el barrio y en la ciudad. A través de dos cortes temporales, se observa un impacto extraordinario de la mortalidad infantil en la Barceloneta, que alcanza unas dimensiones muy por encima de las medias de la ciudad. De las defunciones que se produjeron en el barrio marítimo en los años 1840 y 1850, el 53,42\% fueron de individuos que no superaban los 5 años de edad. Para Barcelona, entre los años 1844 y 1847 , lo fueron un 48,10\%. Lejos de converger a lo largo de las siguientes décadas, los datos del año 1879 son aún más discordantes. Mientras que en el global de la ciudad este porcentaje se ha reducido al 38,93\% del total de muertes contabilizadas, en la Barceloneta 
la cifra aumenta ligeramente a un $55,58 \% .{ }^{24}$ La permanencia de un comportamiento natalista en el barrio muy superior al de Barcelona (Tatjer 1995, p. 128) conllevaba una mortalidad infantil superior que se agravaba por las pésimas condiciones higiénicas y sanitarias de la Barceloneta.

\section{La Barceloneta como espacio social disruptivo}

Más allá de la mortalidad ordinaria, el impacto de los brotes epidémicos en la Barceloneta dejó huella a lo largo del siglo XIX. El mayor de estos se produjo entre el verano y el otoño de 1870, con el contagio de la fiebre amarilla a través de varios marineros extranjeros que llegan al puerto de la ciudad. La Barceloneta, puerta de entrada de la enfermedad, fue aislada por las autoridades y finalmente sus moradores desalojados. La gestión de la crisis implicó una reflexión entre las élites intelectuales y políticas de la ciudad sobre las condiciones que presentaba el barrio. Estas reflexiones no eran más que la acentuación de una preocupación constante y en aumento que, desde mediados del Ochocientos hasta inicios del siglo XX, situaba al barrio como elemento disruptivo de la ciudad. Un dictamen de la Junta Provincial de Sanidad de junio de 1848 ya destacaba la presencia, en las casas de la Barceloneta, de "multitud de familias mal vestidas y peor alimentadas, hacinadas en un estrecho recinto, a veces sin cocina, ni fregadero". ${ }^{25} \mathrm{El}$ reportaje

24. Como hemos observado en la parte inicial del artículo, estos porcentajes eran ya objeto de preocupación en algunas ciudades británicas de mediados de siglo.

25. Dictamen de la Junta Provincial de Sanidad en el pleito entre Francesc y Antoni Costavidal i Closes y otros propietarios de la Barceloneta contra el Ayuntamiento de 152 alertaba de los riesgos para la salubridad de estas condiciones de vida, así como de la falta de ventilación y la acumulación de aguas sucias en los depósitos subterráneos de sus casas. ${ }^{26}$

El año posterior a la epidemia de fiebre amarilla, la Academia de Medicina y Cirugía de Barcelona transmitía al Ayuntamiento un panorama desolador de la Barceloneta y sus "infelices moradores". La pieza denunciaba la falta de agua, el deterioro del pavimento, el hacinamiento de los vecinos y la "sordidez y cochambre" del barrio, sobre el que había que actuar a través de brigadas de limpieza y de una política de severidad ante aquellos que cometiesen infracciones higiénicas. Las medidas propuestas por la Academia fueron bien acogidas y aprobadas por el consistorio. ${ }^{27}$

$\mathrm{La}$ inquietud generada por las condiciones de habitabilidad y de higiene del barrio fue en aumento. García Faría (1893, pp. 152188) señalaba como principales motivos del hecho que la Barceloneta fuese el distrito con mayores niveles de mortalidad de la ciudad en la década de 1880 los pésimos estados de los alcantarillados y pavimentos de las calles, las malas condiciones de las viviendas y la densidad habitacional. No olvidaba señalar el

Barcelona, de 21 de junio de 1848. Caja 844, expediente 3, f. 152, Arxiu General de la Diputació de Barcelona (AGDB), Barcelona.

26. Dictamen de la Junta Provincial de Sanidad en el pleito entre Francesc y Antoni Costavidal i Closes y otros propietarios de la Barceloneta contra el Ayuntamiento de Barcelona, de 21 de junio de 1848. Caja 844, expediente 3, f. 152. AGDB,.

27. Expediente relativo a la conveniencia de tomar varias medidas para la limpieza y saneamiento de la Barceloneta, del 21 al 29 de julio de 1871. Serie A183 de la Comisión de Gobernación del Ayuntamiento de Barcelona, expediente 4420, Arxiu Municipal Contemporani de Barcelona (AMCB), Barcelona. 
abandono de prácticas higiénicas por parte de los vecinos e incluso la miseria, el vicio y la prostitución que reinaban en algunas de sus calles.

La caracterización de la Barceloneta como espacio social problemático que necesitaba ser controlado y reformado, a través de las preocupaciones higienistas dominantes a lo largo de la segunda mitad de siglo, se sitúa de esta forma como elemento clave en la formación de una imagen exterior sobre este espacio y de la propia actuación de las autoridades sobre él, como anteriormente hemos observado en el caso de algunos barrios de grandes ciudades europeas del siglo XIX. De esta forma, se plasmaba sobre la Barceloneta la creciente necesidad de las élites urbanas de disciplinar los espacios de la ciudad percibidos como problemáticos para los ideales de limpieza, progreso, orden y urbanidad que formaron el núcleo del pensamiento higienista (Campos, 1995; Guereña, 2000; Oliveira Sobrinho, 2013; Quintanas, 2011). La inserción del proyecto higienista en el modelo de ciudad civilizada al que aspiraba el poder político y económico alcanzó, de este modo, los barrios portuarios como la Barceloneta, así como muchos otros espacios considerados degradados socialmente en las grandes urbes del planeta..$^{28}$

La mirada higienista no era, sin embargo, una forma exclusiva de relacionarse con el barrio. El establecimiento de baños de mar en su seno, fenómeno de éxito ascendente a lo largo del siglo, así como los frecuentes paseos por el frente marítimo por parte de los barceloneses, provocaron una relación ambigua entre estos últimos y la Barceloneta: al mismo tiempo,

28. Como ejemplo, véase el caso de la ciudad de São Paulo (Oliveira Sobrinho, 2013). resultaba un espacio social alterizado y un sitio predilecto del ocio cotidiano.

\section{Conclusiones}

En la primera mitad del artículo he trazado un perfil de los planteamientos que desde la historia social y marítima se han desarrollado para abordar aquellas sociedades que viven de su relación con el mar. En el estudio de espacios urbanos concretos de residencia de la población marítima se han señalado sus diferentes rasgos propios en el siglo XIX. A partir de estas consideraciones, he abordado el estudio del barrio de la Barceloneta en el siglo XIX en el contexto de su inserción en una gran ciudad portuaria en proceso de industrialización. Los datos elaborados a partir de diferentes fuentes me han permitido concebir a la Barceloneta de mediados de siglo XIX como una comunidad marítima urbana ya asentada, que agrupaba las clases trabajadores de la economía marítima de la ciudad, así como los sectores artesanales y de pequeño comercio ligados a las crecientes demandas de la construcción y reparación naval y del aprovisionamiento y equipamiento de buques.

E1 análisis de diferentes variables sociodemográficas en la Barceloneta entre mediados y finales del siglo XIX ha permitido vislumbrar un perfil específico del barrio con respecto a la ciudad. Unas ratios sexuales ligeramente masculinizadas respecto a la urbe, una estructura por edades de la población notablemente joven, unas tasas de mortalidad elevadas e inclusos crecientes durante el período y la magnitud sobredimensionada de la mortalidad infantil y de los brotes epidémicos puntuales confirman el elevado grado de 
precariedad de la comunidad y los efectos problemáticos de la industrialización y el cambio tecnológico en la economía marítima. Además, estos resultados permiten insertar a la Barceloneta en el contexto comparativo de los estudios de barrios y ciudades portuarias europeos del siglo XIX, confirmando muchos de los patrones que se han apuntado en la historiografía reciente.

Las consideraciones higiénicas y morales centraron el abordaje por parte de las élites y autoridades urbanas de los problemas de la Barceloneta desde mediados de siglo. Estas trazaron un perfil de la Barceloneta marcado por la ausencia de los valores cívicos que debían regir el comportamiento humano en la ciudad según entendían. De cara a nuevas investigaciones, el análisis detallado de más fuentes cualitativas ha de permitirnos profundizar en la caracterización que de la Barceloneta hicieron elementos ajenos a ella, así como comprender con detalle el rol que las mujeres desempeñaron en ella y la estructura y evolución de los relaciones y jerarquías de género en el seno de la comunidad. Asimismo, futuros estudios deben profundizar en la relación global entre el puerto y la ciudad y sus dinámicas de reciprocidad y discordancia en sus respectivas evoluciones.

$$
\propto \ddot{e} \propto
$$

Recibido: 20-05-2018

Aceptado: 20-09-2019

Publicado: 04-12-2019 


\section{Referencias Bibliográficas}

Alemany, J. (1998). El Port de Barcelona. Barcelona: Lunwers Editores.

Alfaro Zaforteza, C. (2007). La sociedad Nuevo Vulcano, la Armada y los orígenes de la navegación de vapor en España, 1834-1855. Drassana: revista del Museu Maritim, O(15), 74-83. Recuperado de https:// www.raco.cat/index.php/Drassana/issue/view/8541/showToc

Ayuntamiento de Barcelona (1903). Anuario estadístico de la ciudad de Barcelona. Año 1902. Barcelona: Ayuntamiento de Barcelona.

Beaven, B. (2016a). From Jolly Sailor to Proletarian Jack: The Remaking of Sailortown and the Merchant Seafarer in Victorian London. En B. Beaven, K. Bell, y R. James (Eds.), Port Towns and Urban Cultures: International Histories of the Waterfront, c.1700-2000 (pp. 159-178). London: Palgrave Macmillan.

Beaven, B. (2016b). The resilience of sailortown culture in English naval ports, c. 1820-1900. Urban History, 43(1), 72-95. https://doi.org/10.1017/S0963926815000140

Beaven, B., Bell, K., y James, R. (Eds.). (2016). Port Towns and Urban Cultures: International Histories of the Waterfront, c.1700-2000. London: Palgrave Macmillan.

Broeze, F. (1985). Port Cities: The Search for an Identity. Journal of Urban History, 11(2), 209-225.

Broeze, F. (1989). Introduction: Brides of the Sea. En F. Broeze (Ed.), Brides of the sea: port cities of Asia from the 16th-20th centuries (pp. 1-28). Kensington: New South Wales University Press.

Byrne, J. (2016). Hull, Fishing and the Life and Death of Trawlertown: Living the Spaces of a Trawling Port-City. En B. Beaven, K. Bell, y R. James (Eds.), Port Towns and Urban Cultures: International Histories of the Waterfront, c.1700-2000 (pp. 243-264). London: Palgrave Macmillan.

Cabantous, A. (2007). Histoire maritime ou histoire sociale? L'approche des gens de mer. Drassana: revista del Museu Maritim, O(15), 84-96. Recuperado de https:/www.raco.cat/index.php/Drassana/issue/ view/8541/showToc

Campos, R. (1995). La sociedad enferma: Higiene moral en España en la segunda mitad del siglo XIX y principio del XX. Hispania: Revista española de historia, 55(191), 1093-1112.

Carbonell, E., Doñate, M., y Romero, P. (2011). Ser o no ser de mar: Arenys: història, patrimoni i antropologia maritima. Girona: Barcelona: Institut Català de Recerca en Patrimoni Cultural: Documenta Universitaria ; Museu Marítim de Barcelona.

Carreras, A., y Yáñez, C. (1992). El puerto en la era industrial: una síntesis histórica. En J. Clavera y Port Autònom de Barcelona (Eds.), Economía e historia del puerto de Barcelona: tres estudios (pp. 81-157). Barcelona : Madrid: Port Autònom de Barcelona ; Civitas.

Cerdà, I. (1868). Monografía estadística de la clase obrera, en 1856: Espécimen de una estadística funcional de la vida urbana, con aplicación concreta á dicha clase. Madrid: Impr. Española. 
Cochard, N. (2016). Les marins du Havre : gens de mer et société urbaine au XIXe siècle. Presses Universitaires de Rennes.

Coll i Llopis, S. (1755). Breve noticia de la fábrica y construccion del nuevo barrio en la playa de Barcelona, llamado vulgarmente Barceloneta. Barcelona: Imprenta de Teresa Piferrer viuda.

Colomer Codina, G. (1883). Movimiento de la población de Barcelona en el veinteno de 1861-1880: Densidad por calles, casas y habitaciones de la población avecindada en Barcelona en 1882. Barcelona: Establecimiento Tipográfico de los Sucesores de N. Ramírez y Cía.

E1 Consultor (1863). El Consultor: Nueva guia de Barcelona: libro de grande utilidad para los vecinos y forasteros, y sumamente indispensable á todos los que pertenecen á las clases mercantil e industrial. (1863). Barcelona: Establecimiento Tipográfico de Narciso Ramírez.

El Consultor (1857). El Consultor: Nueva guía de Barcelona: obra de gran utilidad para todos los vecinos y forasteros, y sumamente indispensable á los que pertenecen á la clase mercantil é industrial. (1857). Barcelona: Imprenta de la publicidad, a cargo de A. Flotats.

Faue, E. (2000). Community, Class, and Comparison in Labour History and Local History. Labour History, (78), 155-162.

García Domingo, E. (2017). Mundo del trabajo en la marina mercante española (1834-1914). Barcelona: Edicions Universitat Barcelona.

Garcia Espuche, A. (2007). Espais urbans de la gent de mar : Barcelona segles XIV al XVIII. Drassana: revista del Museu Maritim, O(15), 36-53-53. Recuperado de https://www.raco.cat/index.php/Drassana/ issue/view/8541/showToc

García Faría, P. (1893). Saneamiento de Barcelona: Condiciones bigiénicas de la urbe: Su mejoramiento, disminución de la mortalidad de sus habitantes y aumento de la vida media de los mismos: Memoria. Tomo I. Barcelona: Establecimiento Tipográfico de los Sucesores de N. Ramírez y Ca.

Garriga i Roca, M. (1862). Plano Topográfico-Geométrico del puerto y población de la Barceloneta aneja a la capital del antiguo Principado con el proyecto de su reforma y mejora arreglado a la Real Orden de 16 de Enero de 1863 por D. Miguel Garriga y Roca arquitecto del Exmo Ayuntamiento de Barcelona. Barcelona.

Gibb, A., y Lee, R. (2002). Industrialitzation and demographic change. A case study of Glasgow, 18011914. En R. Lawton (Ed.), Population and Society in Western European Port Cities, c.1650-1939 (pp. 37-73). Liverpool: Liverpool University Press.

Gritt, A. (2005). Representations of mariners and maritime communities c.1750-1850. History in Focus, (9). Recuperado de https:/www.history.ac.uk/ihr/Focus/Sea/articles/gritt.html

Guereña, J.-L. (2000). Urbanidad, higiene e higienismo. Areas: Revista internacional de ciencias sociales, (20), 61-72. Recuperado de https://revistas.um.es/areas/issue/view/10781 
Hagmark-Cooper, H. (2003). Women in maritime communities: a socio-historical study of continuity and change in the domestic lives of seafarer's wives in the Aland Islands, from 1930 into the new millenium. Hull: Department of History, The University of Hull.

Heyrman, C. L. (1984). Commerce and culture: the maritime communities of colonial Massachusetts, 1690-1750. New York: Norton.

Honorato, C., de Oliveira, A., y Mantuano, T. (2016). Comunidade Portuária do Rio de Janeiro (18701920). En M.-R. García Hurtado y O. Rey Castelao (Eds.), Fronteras de agua: las ciudades portuarias y su universo cultural (siglos XIV-XXI) (pp. 469-484). Santiago de Compostela: Universidade de Santiago de Compostela.

Ibarz Gelabert, J., y Romero Marín, J. (2009). L'abolició de la Matrícula de Mar i les tasques de càrrega i descàrrega al port de Barcelona, 1868-1874. Barcelona quaderns d'història, (15), 255-270. Recuperado de https://www.raco.cat/index.php/BCNQuadernsHistoria/issue/view/12970/showToc

Instituto Geográfico y Estadístico (1879). Memoria relativa a los resultados obtenidos por la Junta Provincial de Barcelona en el Censo General de la Población de 1877, llevado a cabo por la Dirección General del Instituto Geográfico y Estadístico. Barcelona: Censo de la Población. Junta Provincial de Barcelona.

Junta General de Estadística (1863). Censo de población de España, según el recuento verificado en 25 de diciembre de 1860 por la Junta General de Estadistica. Madrid: Imprenta Nacional.

Kaplan, T. (2003). Ciudad roja, período azul: Los movimientos sociales en la Barcelona de Picasso [(1888-1939)]. Barcelona: Península.

Konvitz,J. W. (1993). Port Cities and Urban History. Journal of Urban History, 19(3), 115-120. Recuperado de https://doi.org/10.1177\%2F009614429301900307

Konvitz, J. W. (1994). The Crises of Atlantic Port Cities, 1880 to 1920. Comparative Studies in Society and History, 36(2), 293-318. Recuperado de https://www.jstor.org/stable/179261

Lawton, R. (2002). The components of demographic change in a rapidly growing port-city: the case of Liverpool in the nineteenth century. En R. Lawton y R. Lee (Eds.), Population and Society in Western European Port Cities, c.1650-1939 (pp. 91-123). Liverpool: Liverpool University Press.

Lawton, R., y Lee, R. (2002). Port development and the demographic dynamics of european urbanitzation. En R. Lawton y R. Lee (Eds.), Population and Society in Western European Port Cities, c.1650-1939 (pp. 1-36). Liverpool: Liverpool University Press.

Lee, R., y Lee, W. R. (1998). The socio-economic and demographic characteristics of port cities: a typology for comparative analysis? Urban History, 25(2), 147-172. https://doi.org/10.1017/S096392680000078X

Lee, R. (2013). The Seafarers' Urban World: A Critical Review. International Journal of Maritime History, 25(1), 23-64. Recuperado de https://journals.sagepub.com/doi/abs/10.1177/084387141302500103 
Llovet i Verdura, J. (1980). La matrícula de mar i la província de Marina de Mataró al segle XVIII. Barcelona: Rafael Dalmau.

López Guallar, P. (1995). La demografia barcelonina entre 1833 i 1865. En Història de Barcelona: volum 6: La ciutat industrial (pp. 95-118). Barcelona: Ajuntament de Barcelona : Enciclopèdia Catalana.

Martínez Shaw, C. (1997). La ciudad y el mar: la ciudad marítima y sus funciones en el Antiguo Régimen. Manuscrits: Revista d'història moderna, (15), 257-278. Recuperado de https://ddd.uab.cat/ $\mathrm{record} / 39556$ ? $\mathrm{ln}=\mathrm{ca}$

Mateo, J. (2000). Sant Feliu de Guíxols a la vigília de sortir de casa. L'Arjau, (37), 7-12.Recuperado de http://arxiumunicipal.guixols.cat/attachments/article/6181/arjau_guixols_arxiu_037_temps_carros_ carretes.pdf

Mateo, J. (2004a). Gente que vive del mar: La génesis y el desarrollo de una sociedad marítima y una comunidad pescadora. Prohistoria: historia, politicas de la historia, (8), 59-86. Recuperado de https:// dialnet.unirioja.es/ejemplar/121562

Mateo, J. (2004b). Las Reales Ordenanzas de Marina y la regulación de la pesca en el Ampurdán durante el siglo XVIII. Annals de l'Institut d'Estudis Empordanesos, (37), 111-150. Recuperado de https://www. raco.cat/index.php/AnnalsEmpordanesos/issue/view/26387/showToc

Monge Martínez, F. (1998). Los estudios sobre historia portuaria: Una perspectiva crítica y metodológica. Hispania: Revista española de historia, 58(198), 307-326. Recuperado de https://dialnet.unirioja.es/ ejemplar/1053

Moreno Rico, J. (2011). El capitán de la marina mercante José Ricarty Giralt (1847-1930): una aproximación a la historia marítima contemporánea de Barcelona. Barcelona: Universitat Politècnica de Catalunya.

Oliveira Sobrinho, A. S. de. (2013). São Paulo e a Ideologia Higienista entre os séculos XIX e XX: A utopia da civilidade. Sociologias, 15(32), 210-235. Recuperado de https://seer.ufrgs.br/sociologias/article/ view/38648

Osterhammel, J., y García, G. (2015). La Transformación del mundo: una historia global del siglo XIX. Barcelona: Critica.

Otero Carvajal, L. E. (2007). La reducción de escala y la narratividad histórica. Cuadernos de Historia Contemporánea, Extra 2, 245-264. Recuperado de https://revistas.ucm.es/index.php/CHCO/issue/ view/CHCO070722

Oustric, G. (2016). Une société originale et variée. En A. Lottin (Ed.), Histoire de Boulogne-sur-Mer: ville d'art et d'bistoire (pp. 271-288). Villeneuve d'Ascq: Presses universitaires du Septentrion.

Oyón, J. L., y Serra, M. (2011). Historia urbana: el espacio no es inocente. Historia Contemporánea, O(39). Recuperado de https://www.ehu.eus/ojs/index.php/HC/article/view/2368/0 
Pascual i Domènech, P. (1991). Desenvolupament de la marina de vapor i crisi de la construcció naval i de la marina mercant catalana. En J. Nadal et al. (Ed.), Història econòmica de la Catalunya contemporània (Vol. III, pp. 299-335). Barcelona: Enciclopèdia Catalana.

Permanyer, L. (2003). La Barceloneta, 250 anys d'història. En Ayuntamiento de Barcelona y Gas Natural (Eds.), La Barceloneta, un quart de millleni =: Barceloneta, un cuarto de milenio (pp. 12-35). Barcelona: CAIA.

Pi y Arimón, A. (1854). Barcelona antigua y moderna. Descripción é historia de esta ciudad desde su fundación hasta nuestros dias (Tomo I). Barcelona: Imprenta y Librería Politécnica de Tomás Gorchs.

Polónia, A. (2000). Reflections on social experiences in maritime communities : the portuguese case in the early modern age : specificity or globalisation of a group profile? Repositório Aberto da Universidade do Porto. Recuperado de http://repositorio-aberto.up.pt/handle/10216/13071

Quintanas, A. (2011). Higienismo y medicina social: Poderes de normalización y formas de sujeción de las clases populares. Isegoría, O(44), 273-284. Recuperado de http://isegoria.revistas.csic.es/index.php/ isegoria/issue/view/44

Reeves, P., Broeze, F., y McPherson, K. (1989). Studying the Asian port city. En F. Broeze (Ed.), Brides of the sea:port cities of Asia from the 16th-20th centuries (pp. 29-53). Kensington: New South Wales University Press.

Riera i Tuèbols, S. (2008). La Indústria de construcció de màquines a Catalunya i els Països Catalans. Catalan Historical Review, (1), 239-250. Recuperado de https:/www.raco.cat/index.php/ CatalanHistoricalReview/article/view/131005

Rodrigo Alharilla, M. (2007). Navieras y navieros catalanes en los primeros tiempos del vapor, 1830-1870. Tst: Transportes, Servicios y telecomunicaciones, (13), 62-92. Recuperado de http://www.tstrevista.com/ sumarios/sumario_13.asp

Saurí, M. (1842). Guía de forasteros en Barcelona: Judicial, gubernativa, administrativa, comercial, artística y fabril. Barcelona: Imprenta y Librería de Manuel Saurí.

Saurí, M. (1849). Manual histórico-topográfico, estadístico y administrativo: Ó sea, Guía general de Barcelona. Barcelona: Imprenta y Librería de Manuel Saurí.

Suárez Bosa, M., y Domínguez Prats, P. (2016). Cultura obrera en el barrio portuario de La Isleta (Las Palmas de Gran Canaria): espacio, conflictividad y aprendizaje. En M.-R. García Hurtado y O. Rey Castelao (Eds.), Fronteras de agua: las ciudades portuarias y su universo cultural (siglos XIV-XXI) (pp. 531545). Santiago de Compostela: Universidade de Santiago de Compostela.

Tanis, F. (2016). The Lost Identity of Izmir. En C. Hein (Ed.), History Urbanism Resilience: Ideas on the Move and Modernisation (pp. 371-380). Delft: TU Delft Open.

Tatjer, M. (1973). La Barceloneta: del siglo XVIII al Plan de la Ribera. Barcelona: Saturno. 
Tatjer, M. (1988). Burgueses, inquilinos y rentistas: mercado inmobiliario, propiedad y morfología en el centro histórico de Barcelona: La Barceloneta, 1753-1982. Madrid: Consejo Superior de Investigaciones Científicas.

Tatjer, M. (1995). L'evolució de la població de Barcelona entre el 1860 i el 1897. En Història de Barcelona: volum 6: La ciutat industrial (pp. 119-150). Barcelona: Ajuntament de Barcelona: Enciclopèdia Catalaana.

Valdaliso, J. M. (2001). Entre el mercado y el Estado: la marina mercante y el transporte marítimo en Espana en los siglos XIX y XX. Tst: Transportes, Servicios y telecomunicaciones, (1), 55-79. Recuperado de http://www.tstrevista.com/sumarios/sumario_01.asp 